\title{
Combined assays for serum carcinoembryonic antigen and microRNA-17-3p offer improved diagnostic potential for stage I/II colon cancer
}

\author{
JINHAI ZHU ${ }^{1}$, HUIMING DONG ${ }^{2}$, QIONG ZHANG ${ }^{2}$ and SHANGWU ZHANG ${ }^{3}$
}

\begin{abstract}
Departments of ${ }^{1}$ Oncological Surgery and ${ }^{2}$ Clinical Pathology, The First Affiliated Hospital of Bengbu Medical College, Bengbu, Anhui 233004; ${ }^{3}$ Department of Emergency Surgery, The First Affiliated Hospital of Soochow University,

Suzhou, Jiangsu 215006, P.R. China
\end{abstract}

Received April 9, 2015; Accepted July 6, 2015

DOI: 10.3892/mco.2015.616

\begin{abstract}
Colorectal cancer is among the leading causes of cancer-related mortality, one of the main reasons for which is the lack of an effective screening method for early-stage disease. The levels of carcinoembryonic antigen (CEA) and microRNA (miR)-17-3p in the serum of 70 patients with stage I/II colon cancer and 70 healthy volunteers were determined, and the diagnostic value of CEA plus miR-17-3p detection for colon cancer was assessed. The levels of CEA were measured by a radioimmunoassay method, and those of miR-17-3p using the reverse transcription-quantitative polymerase chain reaction method. miR-16 was used as the endogenous control, as it displayed high stability, high abundance and low variability in the analyzed serum samples. The receiver operating characteristic (ROC) curve analysis indicated the potential diagnostic value of the two markers and the area under the ROC curve (AUC) for CEA and miR-17-3p was 0.719 (95\% CI: 0.658-0.843) and 0.807 (95\% CI: 0.748-0.906), respectively. At a threshold of $9.6 \mathrm{ng} / \mathrm{ml}$ for CEA, the optimal sensitivity and specificity were 74.6 and $84.3 \%$, respectively, in discriminating colon cancer patients from healthy controls. At a threshold of 2.98 for miR-17-3p, the sensitivity and the specificity were 83.6 and $72.9 \%$, respectively. A combined ROC analysis using CEA and miR-17-3p revealed an AUC of 0.929 (95\% CI: 0.834-0.978) with a sensitivity of $96.4 \%$ and a specificity of $95.7 \%$ in discriminating colon cancer patients from healthy controls. In conclusion, both CEA and miR-17-3p were highly expressed in the serum of our series of colon cancer patients. CEA plus miR-17-3p detection significantly increased the sensitivity and specificity in discriminating stage I/II colon cancer patients from healthy controls. Therefore, combined
\end{abstract}

Correspondence to: Dr Shangwu Zhang, Department of Emergency Surgery, The First Affiliated Hospital of Soochow University, 188 Shizhi Road, Suzhou, Jiangsu 215006, P.R. China

E-mail: 1461386112@qq.com

Key words: colon cancer, microRNA-17-3p, carcinoembryonic antigen, combination, diagnosis detection of serum CEA and miR-17-3p levels may have the potential to become a new laboratory method for the early clinical diagnosis of colon cancer.

\section{Introduction}

Colorectal cancer (CRC) is the third most common type of cancer worldwide and one of the leading causes of cancer-related mortality (1). Lack of an effective method for screening early-stage colon cancer is an important reason for its high mortality rate. Patients with early-stage colon cancer have a favorable outcome with treatment, with the 5-year survival rates exceeding $70 \%$ for stage I/II colon cancer $(2,3)$. Colonoscopy has been used for screening early-stage colon cancer for several decades as a first choice; however, due to its invasive character, it is associated with low compliance in the clinical setting. Therefore, cost-effective and non-invasive biomarkers with high sensitivity and specificity are needed to enable early-detection of stage I/II colon cancer.

Recently, several studies have focused on the role of various biomarkers in early-stage cancer progression and diagnosis (4-6). Carcinoembryonic antigen (CEA), a well-known tumor marker, is found at low levels in the serum of heathy adults, but at higher levels in colon cancer patients (4). However, CEA is not a reliable independent marker for diagnosing cancer or for screening tests to detect early-stage cancer (7), as its levels are also increased in certain non-neoplastic diseases, including ulcerative colitis, pancreatitis, cirrhosis and hypothyroidism (5). Hence, there remains a high demand for novel and reliable biomarkers with high specificity.

An increasing number of recent studies have focused on the potential of microRNAs (miRNAs) for assessing colon cancer diagnosis or progression. miRNAs have been reported to play an important role in a number of important cellular processes, and may be involved in colon carcinogenesis through deregulation of oncogenes or tumor suppressor genes (8). Several studies have recently identified miRNAs expressed at different levels in the serum of cancer patients compared with healthy controls (9-12). miRNAs have been suggested as potential reliable biomarkers for tumor screening $(13,14)$ due to their extremely stable nature and the fact that they may be 
easily measured by common laboratory methods. In 2009 , $\mathrm{Ng}$ et al (15) analyzed 95 miRNAs and identified miRNA (miR)-17-3p as exhibiting the most significant overexpression in the plasma and tumor tissues of colon cancer patients. However, a later study found no significant differences between miR-17-3p levels in the serum of CRC patients and heathy controls (16).

The aim of the present study was to investigate whether CEA and miR-17-3p are elevated in the serum of stage I/II colon cancer patients and assess their potential as novel biomarkers for this type of cancer.

\section{Subjects and methods}

Subjects. Serum samples were obtained from 70 colon cancer patients who were diagnosed at the First Affiliated Hospital of Soochow University (Suzhou, China) and the First Affiliated Hospital of Bengbu Medical College, (Bengbu, China) between 2011 and 2013. An additional 70 serum samples were obtained from healthy control individuals with no previous history of any cancer, who were recruited from the same hospitals. All the subjects were of the same ethnicity (Chinese Han). The clinical and pathological characteristics, including gender, age and clinical stage, were recorded for colon cancer patients and are summarized in Table I. The patients were selected retrospectively on the basis of clinical characteristics to include only patients with stage I/II colon cancer. Informed consent was obtained from each subject, and the study was approved by the Ethics Committee of the First Affiliated Hospital of Soochow University, Suzhou, China.

Sample collection. Blood samples were obtained immediately following diagnosis and prior to the patients receiving any oncological treatment. Blood was processed for serum within $2 \mathrm{~h}$ and stored at $-80^{\circ} \mathrm{C}$. The median time from storage to endpoint analysis was 12 months. Serum was obtained by centrifugation at $12,000 \mathrm{x}$ g for $10 \mathrm{~min}$ at $4^{\circ} \mathrm{C}$.

Serum CEA determination. CEA was assayed with a immunoradiometric assay (Beijing Dongya Biotechnology; Beijing, China) and the threshold for a positive result was $5 \mathrm{ng} / \mathrm{ml}$. The assay was performed according to the manufacturer's instructions.

RNA isolation, reverse transcription (RT) and quantitative-polymerase chain reaction ( $q P C R)$. Total RNA enriched for small RNAs was isolated from $250 \mu 1$ aliquots of serum using Qiagen miRNeasy Mini kits (Qiagen GmbH, Hilden, Germany) according to the manufacturer's modified protocol. For each sample, $1.25 \mu \mathrm{l}$ of $0.8 \mu \mathrm{g} / \mu \mathrm{l}$ MS2 RNA carrier was added to $800 \mu \mathrm{l}$ of QIAzol solution. The extracted RNA was eluted in $30 \mu \mathrm{l}$ of preheated elution solution, and the concentration and purity of the RNA were estimated spectrophotometrically (A260/280>2.0; A260/230>1.8) using a NanoDrop ND-1000 spectrophotometer (Thermo Fisher Scientific, Wilmington, DE, USA). The RNA samples were either further processed immediately or stored at $-80^{\circ} \mathrm{C}$.

Complementary DNA was synthesized from total RNA using gene-specific primers according to the TaqMan MicroRNA Assay protocol (Applied Biosystems, Foster City,
Table I. Summary of expression levels of CEA and miR-17-3P detected in serum of colon cancer patients and healthy donors expressed as median and interquartile range.

\begin{tabular}{lccc}
\hline Variables & $\begin{array}{c}\text { Colon cancer } \\
\text { patients } \\
(\mathrm{n}=70)\end{array}$ & P-value $^{\mathrm{b}}$ \\
\hline Age (years $)$ & & & \\
Mean \pm SD & $47.34 \pm 10.13$ & $49.58 \pm 12.62$ & \\
Gender, no. & & 48 & \\
Male & 40 & 22 & \\
Female & 30 & 14 & \\
Stage, no. & & 56 & 0.032 \\
I & & & \\
II & & $9.79 \pm 3.43$ & 0.01 \\
CEA (ng/ml) & $1.76 \pm 0.88$ & $3.25 \pm 2.69$ & \\
Mean \pm SD & & & \\
miR-17-3p & & & \\
Mean \pm SD & $0.61 \pm 0.48$ & & \\
\hline
\end{tabular}

${ }^{a}$ The values for miR-17-3p were multiplied by 1,000 . ${ }^{\mathrm{b}}$ The Mann-Whitney U test was used to determine statistical significance. CEA, carcinoembryonic antigen; SD, standard deviation.

CA, USA). For RT reactions, $10 \mathrm{ng}$ of RNA sample, $50 \mathrm{nM}$ of stem-loop RT primer, 1X RT buffer, $0.25 \mathrm{mM}$ each of dNTPs, $3.33 \mathrm{U} / \mu 1$ MultiScribe RT and $0.25 \mathrm{U} / \mu 1 \mathrm{RNase}$ inhibitor were used. The RT mixtures $(15 \mu \mathrm{l})$ were incubated for $30 \mathrm{~min}$ at $16^{\circ} \mathrm{C}, 30 \mathrm{~min}$ at $42^{\circ} \mathrm{C}$ and $5 \mathrm{~min}$ at $85^{\circ} \mathrm{C}$, and then maintained at $4^{\circ} \mathrm{C}$ (PTC-200 Thermal Cycler; MJ Research, Inc., Waltham, MA, USA). qPCR was performed using the 7500 Real-Time PCR system (Applied Biosystems). The 20- $\mu$ l PCR reaction mixture included $1.33 \mu \mathrm{l}$ of RT product, $1 \mathrm{X}$ TaqMan Universal PCR master mix, and $1 \mu \mathrm{l}$ of primer and probe mix from the TaqMan MicroRNA Assay kit (Applied Biosystems). The qPCR reactions were incubated in a 96 -well optical plate at $95^{\circ} \mathrm{C}$ for $10 \mathrm{~min}$, followed by 40 cycles at $95^{\circ} \mathrm{C}$ for $15 \mathrm{sec}$ and at $60^{\circ} \mathrm{C}$ for $10 \mathrm{~min}$.

Data normalization and statistical analysis. The cycle threshold $(\mathrm{Ct})$ values were calculated using SDS 2.0.1 software (Applied Biosystems) using the default threshold settings (automatic baseline, threshold 0.2). All the qPCR reactions were run as triplicates and the mean $\mathrm{Ct}$ and standard deviation values were calculated. The average expression levels of miR-17-3p were normalized using miR-16 (Applied Biosystems) as a reference gene and subsequently the $2^{-\Delta \Delta \mathrm{CT}}$ method was applied. The Mann-Whitney U test was used to compare the serum CEA and miRNA expression between the different groups, as it is a non-parametric test of the null hypothesis that two samples come from the same population against an alternative hypothesis, particularly that one population tends to exhibit larger values compared with the other, and may be applied on unknown distributions. Receiver operating characteristic (ROC) curves and the area under the ROC curve (AUC) assessed the feasibility of using serum miRNA as a 


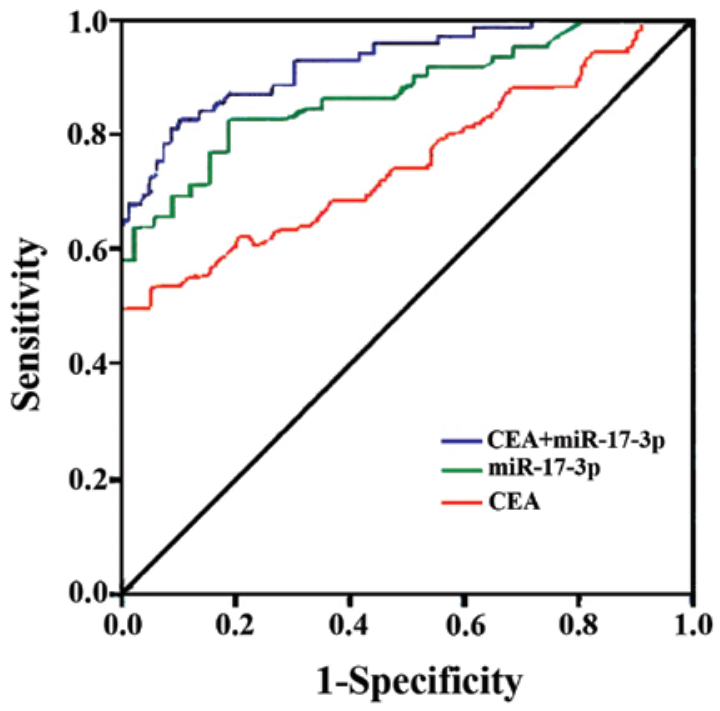

Figure 1. ROC curve analysis of serum CEA and microRNA (miR)-17-3p levels. At a threshold of $9.6 \mathrm{ng} / \mathrm{ml}$ for CEA, the optimal sensitivity and specificity were 74.6 and $84.3 \%$ in separating colon cancer patients from healthy controls. At a threshold of 2.98 for miR-17-3p, the sensitivity and the specificity were 83.6 and $72.9 \%$, respectively. Combined ROC curve analysis using CEA and miR-17-3p revealed an AUC of 0.929 (95\% CI: 0.834-0.978) with a sensitivity of $96.4 \%$ and a specificity of $95.7 \%$ in discriminating colon cancer patients from healthy controls. CEA, carcinoembryonic antigen; ROC, receiver operating characteristic AUC, area under the ROC curve CI, confidence interval.

diagnostic tool for colon cancer. The Youden index determined the threshold for the plasma miRNA concentrations. All the tests were two-sided and $\mathrm{P}<0.05$ was considered to indicate statistically significant differences. The statistical analysis was conducted using SPSS 16.0 software (SPSS Ltd., Woking, Surrey, UK) and the graphs were generated with GraphPad Prism 5.0 (GraphPad Software Inc., San Diego, CA, USA).

\section{Results}

Serum sample examination. To evaluate the diagnostic value of miR-17-3p and CEA that were identified in previous studies $(16,17)$, serum samples of 70 stage I/II colon cancer patients and 70 healthy controls were examined by radioimmunoassay and RT-qPCR, respectively. miR-16 was used as the endogenous control, as it displayed high stability, high abundance and low variability in analyzed serum samples.

$C E A$ and miR-17-3p serum levels. Using the Mann-Whitney U test, the CEA levels were found to be significantly elevated in the serum of stage I/II colon cancer patients compared with healthy controls $(\mathrm{P}<0.01)$. Moreover, miR-17-3p also exhibited significantly different expression levels $(\mathrm{P}<0.001)$ between colon cancer patients and controls (Fig. 1). Additionally, the ROC curve analysis indicated their potential diagnostic values: The AUCs for CEA and miR-17-3p were 0.719 (95\% CI: 0.658-0.843) and 0.807 (95\% CI: 0.748-0.906), respectively. At a threshold of $9.6 \mathrm{ng} / \mathrm{ml}$ for CEA, the optimal sensitivity and specificity were 74.6 and $84.3 \%$ in separating colon cancer patients from normal controls. At a threshold of 2.98 for miR-17-3p, the sensitivity and the specificity were 83.6 and $72.9 \%$, respectively. When the test results for CEA and miR-17-3p were considered jointly, the ROC analysis revealed an AUC of 0.929 (95\% CI: 0.834-0.978) with a sensitivity of $96.4 \%$ and specificity $95.7 \%$ in discriminating colon cancer patients from healthy controls.

\section{Discussion}

Several studies recently reported that CEA and miR-17-3p individually may be used as potential markers for the diagnosis of CRC (16) and pancreatic cancer (17) by its differentiated expression level in the plasma or serum. In this study, we analyzed the levels of CEA and miR-17-3p in the serum of stage I/II colon cancer patients and healthy controls. Compared with healthy controls, both CEA and miR-17-3p were overexpressed in the serum of colon cancer patients. Moreover, the results of the ROC analysis using a combination of CEA and miR-17-3p revealed an AUC of 0.927 with a sensitivity of $93 \%$ and a specificity of $94.7 \%$ in discriminating colon cancer patients from healthy controls.

CEA is one of the most widely used tumor markers, and has been found to be elevated in the plasma or serum of patients with various types of cancer, including CRC, pancreatic, lung and breast cancer $(17,18)$. Based on the high expression of CEA in colon cancer, the diagnosis of colon cancer was improved via CEA level detection $(19,20)$. However, CEA expression has also been reported to be increased in certain non-cancerous conditions, such as ulcerative colitis, pancreatitis, cirrhosis and hypothyroidism $(5,21)$. Therefore, CEA alone may not offer a reliable screening test for early cancer detection (7). Our results confirmed that CEA is elevated in the serum of patients with stage I/II colon cancer compared with healthy controls, and demonstrated that the combined ROC analysis using both CEA and miR-17-3p may significantly increase the sensitivity and specificity of CEA; thus, CEA may be a useful component in combined biomarker detection due to its high sensitivity.

miRNAs play an important role in carcinogenesis, they have been assessed as diagnostic biomarkers $(13,22)$ and prognostic factors (23) and have been used in the therapeutic strategies for various cancers (13). Various miRNAs have been found to be present at reduced or elevated levels in the serum or plasma of colon cancer patients $(12,22,24)$. Considering miR-17-3p levels in the serum of colon cancer patients, previous investigations have yielded contradictory results. In 2009, $\mathrm{Ng}$ et al identified an elevation of miR-17-3p levels in the plasma of CRC patients (15). Our results support this finding, as we found elevated miR-17-3p levels in the serum of early-stage colon cancer patients. However, in 2013, Faltejskova et al reported that there was no significant difference in the serum levels of miR-17-3p between CRC patients and healthy controls (16). As we analyzed miR-17-3p expression using the same protocol as that used in the Faltejskova et al study, we also used miR-16 as control to evaluate the expression. A potential explanation for these contradictory results may be that the study of $\mathrm{Ng}$ et al and our study were performed on Asian populations, whereas the study of Faltejskova et al included a Caucasian population.

Combining detection of different biomarkers has been reported to be a useful strategy in several studies $(12,13,17,22)$, as it increases the sensitivity and specificity of each biomarker. In the present study, CEA exhibited high specificity and low 
sensitivity, while miR-17-3p exhibited the opposite properties. In a combined ROC analysis using the two markers, both sensitivity and specificity reached a relatively high value in discriminating early-stage colon cancer patients from healthy controls. To date, no reliable independent biomarker has been established for early-stage colon cancer screening. Our results suggest that the combination of CEA and miR-17-3p serum assays may offer a useful tool for the detection of early-stage colon cancer detection.

In conclusion, the combined detection of CEA and miR-17-3p may prove to be of clinical value for the early screening of colon cancer. Combined CEA and miR-17-3p detection was found to significantly increase sensitivity and specificity in early-stage colon cancer diagnosis. Therefore, the combined detection of serum CEA and miR-17-3p may have the potential to become a new laboratory method for the clinical diagnosis of colon cancer.

\section{References}

1. Jemal A, Siegel R, Ward E, Hao Y, Xu J and Thun MJ: Cancer statistics, 2009. CA Cancer J Clin 59: 225-249, 2009.

2. Wolpin BM, Meyerhardt JA, Mamon HJ and Mayer RJ: Adjuvant treatment of colorectal cancer. CA Cancer J Clin 57: 168-185, 2007.

3. Storli KE, Søndenaa K, Bukholm IR, Nesvik I, Bru T, Furnes B, Hjelmeland B, Iversen KB and Eide GE: Overall survival after resection for colon cancer in a national cohort study was adversely affected by TNM stage, lymph node ratio, gender, and old age. Int J Colorectal Dis 26: 1299-1307, 2011.

4. StanfordCancerCenter:Cancerdiagnosis.Informationaboutcancer. https://stanfordhealthcare.org/medical-clinics/cancer-center.html. Accessed October 15, 2008

5. Schimanski CC, Frerichs K, Rahman F, Berger M, Lang H, Galle PR, Moehler M and Gockel I: High miR-196a levels promote the oncogenic phenotype of colorectal cancer cells. World J Gastroenterol 15: 2089-2096, 2009.

6. Luo X, Stock C, Burwinkel B and Brenner H: Identification and evaluation of plasma microRNAs for early detection of colorectal cancer. PLoS One 8: e62880, 2013.

7. Duffy MJ, van Dalen A, Haglund C, Hansson L, Klapdor R, Lamerz R, Nilsson O, Sturgeon C and Topolcan O: Clinical utility of biochemical markers in colorectal cancer: European Group on Tumour Markers (EGTM) guidelines. Eur J Cancer 39: 718-727, 2003.

8. Zhang B, Pan X, Cobb GP and Anderson TA: microRNAs as oncogenes and tumor suppressors. Dev Biol 302: 1-12, 2007.

9. Bandrés E, Cubedo E, Agirre X, Malumbres R, Zárate R, Ramirez N, Abajo A, Navarro A, Moreno I, Monzó M, et al: Identification by real-time PCR of 13 mature microRNAs differentially expressed in colorectal cancer and non-tumoral tissues. Mol Cancer 5: 29, 2006.

10. Yamamichi N, Shimomura R, Inada K, Sakurai K, Haraguchi T, Ozaki Y, Fujita S, Mizutani T, Furukawa C, Fujishiro M, et al: Locked nucleic acid in situ hybridization analysis of miR-21 expression during colorectal cancer development. Clin Cancer Res 15: 4009-4016, 2009.
11. Lanza G, Ferracin M, Gafà R, Veronese A, Spizzo R, Pichiorri F, Liu CG, Calin GA, Croce CM and Negrini M: mRNA/microRNA gene expression profile in microsatellite unstable colorectal cancer. Mol Cancer 6: 54, 2007.

12. Schepeler T, Reinert JT, Ostenfeld MS, Christensen LL, Silahtaroglu AN, Dyrskjøt L, Wiuf C, Sørensen FJ, Kruhøffer M, Laurberg S, et al: Diagnostic and prognostic microRNAs in stage II colon cancer. Cancer Res 68: 6416-6424, 2008.

13. Chen X, Ba Y, Ma L, Cai X, Yin Y, Wang K, Guo J, Zhang Y, Chen J, Guo X, et al: Characterization of microRNAs in serum: A novel class of biomarkers for diagnosis of cancer and other diseases. Cell Res 18: 997-1006, 2008.

14. Etheridge A, Lee I, Hood L, Galas D and Wang K: Extracellular microRNA: A new source of biomarkers. Mutat Res 717: 85-90, 2011.

15. Ng EK, Chong WW, Jin H, Lam EK, Shin VY, Yu J, Poon TC, $\mathrm{Ng}$ SS and Sung JJ: Differential expression of microRNAs in plasma of patients with colorectal cancer: A potential marker for colorectal cancer screening. Gut 58: 1375-1381, 2009.

16. Faltejskova P, Bocanek O, Sachlova M, Svoboda M, Kiss I, Vyzula R and Slaby O: Circulating miR-17-3p, miR-29a, miR-92a and miR-135b in serum: Evidence against their usage as biomarkers in colorectal cancer. Cancer Biomark 12: 199-204, 2012.

17. Chen Y, Gao SG, Chen JM, Wang GP, Wang ZF, Zhou B, Jin CH, Yang YT and Feng XS: Serum CA242, CA199, CA125, CEA, and TSGF are biomarkers for the efficacy and prognosis of cryoablation in pancreatic cancer patients. Cell Biochem Biophys, 2014.

18. Hammarström S: The carcinoembryonic antigen (CEA) family: Structures, suggested functions and expression in normal and malignant tissues. Semin Cancer Biol 9: 67-81, 1999.

19. Monzo M, Navarro A, Bandres E, Artells R, Moreno I, Gel B, Ibeas R, Moreno J, Martinez F, Diaz T, et al: Overlapping expression of microRNAs in human embryonic colon and colorectal cancer. Cell Res 18: 823-833, 2008.

20. Shi B, Sepp-Lorenzino L, Prisco M, Linsley P, deAngelis T and Baserga R: Micro RNA 145 targets the insulin receptor substrate- 1 and inhibits the growth of colon cancer cells. J Biol Chem 282: 32582-32590, 2007

21. Maestranzi S, Przemioslo R, Mitchell H and Sherwood RA: The effect of benign and malignant liver disease on the tumour markers CA19-9 and CEA. Ann Clin Biochem 35: 99-103, 1998.

22. Wang Q, Huang Z, Ni S, Xiao X, Xu Q, Wang L, Huang D, Tan C, Sheng $\mathrm{W}$ and Du X: Plasma miR-601 and miR-760 are novel biomarkers for the early detection of colorectal cancer. PLoS One 7: e44398, 2012.

23. Cheng H, Zhang L, Cogdell DE, Zheng H, Schetter AJ, Nykter M, Harris CC, Chen K, Hamilton SR and Zhang W: Circulating plasma miR-141 is a novel biomarker for metastatic colon cancer and predicts poor prognosis. PLoS One 6: e17745, 2011.

24. Schetter AJ, Leung SY, Sohn JJ, Zanetti KA, Bowman ED, Yanaihara N, Yuen ST, Chan TL, Kwong DL, Au GK, et al: MicroRNA expression profiles associated with prognosis and therapeutic outcome in colon adenocarcinoma. JAMA 299: 425-436, 2008 